\title{
Analysis and comparison of numerical algorithms for finding the GCD of certain types of polynomials in the Chebyshev basis
}

\author{
Siti Nor Asiah binti Isa, Nor'aini Aris, Ahmad Zharif Salami bin Mohd Taha* \\ Department of Mathematical Science, Faculty of Science, Universiti Teknologi Malaysia, Skudai 81310, Johor, Malaysia \\ *Corresponding author: snasiahisa@gmail.com,noraini@utm.my
}

\section{Article history}

Received 18 February 2017

Accepted 27 Mac 2017

\begin{abstract}
This research investigates on the numerical methods for computing the greatest common divisors (GCD) of two polynomials in the orthogonal basis without having to convert to the power series form Previous implementations were conducted using the Gauss Elimination with partial pivoting (GEPP) and QR Householder algorithms, respectively. This work proceeds to seek for a better approximate solution by comparing the results of the implementations with the QR with column pivoting (QRCP) algorithm. The results reveal that QRCP is as competent as the GEPP algorithm, up to a certain degree, giving a reasonably good approximate solution. It is also found that normalizing the columns of the associated coefficient matrix slightly reduces the condition number of the matrix but has no significant effect on the GCD solutions when applying the GEPP and QR Householder algorithms. However equilibration of the columns by computing its $\infty$-norm is capable to improve the solution when QRCP is applied. Comparing the three algorithms on some test problems, QR Householder outperforms the rest and is able to give a good approximate solution in the worst case condition when the smallest element of the matrix is 1 , the entries ranging up to 15 digits integers.
\end{abstract}

Keywords: Greatest common divisor (GCD), Gauss elimination, QR decomposition, over determined systems, normalization

(c) 2017 Penerbit UTM Press. All rights reserved

\section{INTRODUCTION}

Problem arises in image processing [1, 2], control theory [3], and the calculation of multiple roots of a polynomial [4] require the computation of the greatest common divisor (GCD) of two poynomials. Generally, polynomials in the orthogonal basis may be better conditioned than that of the power series form when finding polynomial roots. The representation of polynomials in the orthogonal basis is very useful in the numeric computations due to its wellconditioned property which when applied using appropriate numerical methods give better approximation to the root finding problem.

The theory and applications of polynomials in the generalized form have been well studied by Barnett $[5,6,7]$ as proposed in several of his works. Aris [8] and Ahmad et al. [9] has implemented the theories and analytical results in Barnett $[5,6,7]$ by presenting a new symbolic algorithm for computing the GCD of polynomials in the orthogonal basis, in particular the Legendre and Chebyshev polynomial basis. The work have applied the modular homomorphic image scheme so as to avoid multiprecision operations involving very large integers and rational numbers in the coefficients of the polynomials represented in its orthogonal form. An improved algorithm that combines the modular approach with rational number computations at different stages of the algorithm had also been shown in Aris [10]. From these works, the computations were succesfully done in the orthogonal polynomial basis, rather then converting to the power series form. Thus, in the exact computation environment, the theoretical and emprical aspects of finding the GCD of certain class of polynomials in the orthogonal basis using the theorem of Barnett were well achieved and presented.
The question of whether it is also possible to apply appropriate numerical computational tools for solving the GCD or other related problems involving orthogonal basis polynomials, without having to convert to the power series basis remains a challenging task. The comrade matrix approach reduces to a linear algebra problem of finding the rank and solving overdetermined systems of linear equations. The motivation to proceed from previous works in Aris [8] and Ahmad et al. [9] using the comrade matrix approach under the floating point environment is not only for solving the GCD, but also to further investigate on the strength and limitations of the chosen numerical methods of solving the corresponding systems of equations. The main task is to develop a suitable technique that can solve the problem when the entries of the coefficient matrix involve very large integers with a large disparity in the magnitude of the smallest and largest entries of the matrix.

In this paper, the task of computing the GCD of two polynomials relative to an orthogonal basis, in particular the shifted Chebyshev polynomial basis, using the comrade matrix approach is investigated. Performing computations in the floating point environment, the ultimate aim of the research is to propose appropriate numerical methods for solving the problem. As in Isa et. al. [11], apart from getting a good approximate solution, this research intends to investigate how the input data from the polynomial coefficients or the coefficient matrix entries can affect the accuracy of the solutions. Therefore, besides the stability criterion of the chosen numerical methods, the conditioning property of the coefficient matrix is also considered.

The method of computing the GCD of two polynomials $a(x)$ and $b(x)$ based on comrade matrix approach leads to solving 
corresponding systems of linear equations and the work done in two stages. In particular, the degree of GCD of the polynomials which equal to the rank loss of the coefficient matrix is initially determined, after which, the coefficients of the GCD are obtained from solving the associated systems. Considering the conditioning property of coefficient matrix, appropriate stable methods are needed to solve the systems of equations which is constructed from the comrade matrix. In this paper, results using the well-known Gauss elimination method with partial pivoting (GEPP), the QR Householder decomposition and the QR Householder with column pivoting (QRCP) algorithm for solving systems of linear equations are implemented to find the GCD of certain class of polynomials and the results presented.

\section{THE COMRADE MATRIX APPROACH}

The comrade matrix was introduced as the analogue of the companion matrix when a polynomial is expressed in terms of a basis set of orthogonal polynomials. It is shown that the determination of finding the greatest common divisor (GCD) of two or more polynomials can be extended to the case of generalized polynomials by using the comrade matrix (Barnett, 1984). Let

$$
p_{i}(x)=\sum_{i=0}^{n} p_{i j} x_{j}
$$

be a set of real orthogonal polynomials defined by the relationship of

$$
\begin{aligned}
& p_{0}(x)=1 \\
& p_{1}(x)=\alpha_{0} x+\beta_{0} \\
& p_{i+1}(x)=\left(\alpha_{i}(x)+\beta_{i}(x)\right) p_{i}(x)-\gamma_{i} p_{i-1}(x)
\end{aligned}
$$

for $i=1,2,3, \ldots, n-1$ with $\alpha_{i}>0, \beta_{i}>0, \gamma_{i}>0$. Any given $n$th degree univariate polynomial in $x$ can be uniquely expressed as a linear combination of the set $p_{0}(x), p_{1}(x), \ldots, p_{n}(x)$.

Consider two arbitrary polynomials $\tilde{a}(x)=\tilde{a}_{0}+\tilde{a}_{1} x+\tilde{a}_{n} x^{n}$ and $\tilde{b}(x)=\tilde{b}_{0}+\tilde{b}_{1} x+\cdots+\tilde{b}_{m} x^{m}$ with coefficient over a field can be written as

$$
\begin{aligned}
& a(x)=a_{0} p_{0}(x)+a_{1} p_{1}(x)+\cdots+a_{n} p_{n}(x) . \\
& b(x)=b_{0} p_{0}(x)+b_{1} p_{1}(x)+\cdots+b_{m} p_{m}(x) .
\end{aligned}
$$

With no loss of generality, let $a_{n}=1$ and $m<n$. If the values of $\alpha_{i}, \beta_{i}, \gamma_{i}$ for $i=0,1, \ldots, n-1$ are known, the comrade matrix for $a(x)$ is an $n \times n$ matrix given by

$$
\left[\begin{array}{cccccccc}
\frac{-\beta_{0}}{\alpha_{0}} & \frac{1}{\alpha_{0}} & \cdots & 0 & \cdots & \cdots & \cdots & 0 \\
\frac{\gamma_{1}}{\alpha_{1}} & \frac{-\beta_{1}}{\alpha_{1}} & \frac{1}{\alpha_{1}} & 0 & \cdots & \cdots & \cdots & 0 \\
0 & \frac{\gamma_{2}}{\alpha_{2}} & \frac{-\beta_{2}}{\alpha_{2}} & \frac{1}{\alpha_{2}} & \cdots & \cdots & \cdots & 0 \\
\vdots & \vdots & \vdots & \vdots & \cdots & \cdots & \cdots & \vdots \\
0 & 0 & 0 & 0 & \cdots & \cdots & \cdots & \frac{1}{\alpha_{n-2}} \\
\frac{-a_{0}}{a_{n} \alpha_{n-1}} & \cdots & \cdots & \cdots & \cdots & \frac{-a_{n-3}}{a_{n} \alpha_{n-1}} & \frac{-a_{n-2}+a_{n} \gamma_{n-1}}{a_{n} \alpha_{n-1}} & \frac{-a_{n-1}-a_{n} \beta_{n-1}}{a_{n} \alpha_{n-1}}
\end{array}\right]
$$

\section{Constructing the system of equations}

\section{If $A$ is the comrade matrix given as above, define}

$$
b(A)=b_{0} I+b_{1} p_{1}(A)+\cdots+b_{m} p_{m}(A) .
$$

The rows of $b(A)$ are respectively $r_{0}=\left[b_{0}, b_{1}, \ldots, b_{m}, \ldots, b_{n-1}\right], r_{1}=$ $r_{0} p_{1}(A), \ldots, r_{n-1}=r_{0} p_{n-1}(A)$. Using the recurrence relation defined in (2), we obtain the rows in term of the comrade matrix and recurrence relation such that:

$$
\begin{aligned}
& r_{0}=\left(b_{0}, b_{1}, \ldots, b_{m}, 0 \ldots, 0\right), \\
& r_{1}=r_{0}\left(\alpha_{0} A\right), \\
& r_{i}=r_{i-1}\left(\alpha_{i-1} A+\beta_{i-1} I\right)-\gamma_{i-1} r_{i-2},
\end{aligned}
$$

for $i=2, \ldots, n$. It is known [6] that $k=n-\operatorname{rank}[b(A)]$ is the degree of the GCD of $a(x)$ and $b(x)$ such that

$$
d(x)=d_{0} p_{0}(x)+d_{1} p_{1}(x)+\cdots+p_{k}(x) .
$$

Theorem 1 For $i=1,2, \ldots, n$, let $c_{i}$ be the $i_{t h}$ column of $b(A)$. The columns $c_{k+1}, \ldots, c_{n}$ are linearly independent and the coefficients $d_{0}, \ldots, d_{k-1}$ in (7) are given by

$$
c_{i}=d_{i-1} c_{k+1}+\sum_{k+2}^{n} x_{i j} c_{j} \quad i=1,2, \ldots, k \text { for some } x_{i j}
$$

The $k$-system of equations in (8) is described by the augmented matrix

(9)

$$
\left(\begin{array}{llllllllllllll}
c_{k+1} & \vdots & c_{k+2} & \vdots & \ldots & \vdots & c_{n} & \| & c_{1} & \vdots & \ldots & \vdots & c_{k}
\end{array}\right)
$$

which is

$$
\left(\begin{array}{cccc}
c_{1, k+1} & c_{1, k+2} & \ldots & c_{1, n} \\
c_{2, k+1} & c_{2, k+2} & \ldots & c_{2, n} \\
\vdots & \vdots & \ldots & \vdots \\
c_{n, k+1} & c_{n, k+2} & \ldots & c_{n, n}
\end{array}\right)\left(\begin{array}{c}
x_{i, k+1} \\
x_{i, k+2} \\
\vdots \\
x_{i n}
\end{array}\right)=\left(\begin{array}{c}
c_{1 i} \\
c_{2 i} \\
\vdots \\
c_{n i}
\end{array}\right)
$$

(10)

for each $i=1,2, \ldots, k$ and $x_{i, k+1}=d_{i-1}$.

The computation of the rank of $b(A)$ and the coefficient of GCD, can be computed simultaneously from (9) and (10). The column of matrix $b(A)$ is rearranged to produce a new matrix so that the $j^{\text {th }}$ column of $b(A)$ is the $n-(j-1)^{t h}$ column of the new matrix, say $L^{(0)}$ i.e:

$$
l_{n-(j-1)}^{0}=c_{j} \text { for } j=1,2, \ldots, n .
$$

Reducing $L^{(0)}$ to upper row echelon form by $s$ steps gives the matrix

$$
L^{(s)}=\left(\begin{array}{cccccccc}
l_{11}^{(s)} & l_{12}^{(s)} & \ldots & l_{1 r}^{(s)} & \vdots & l_{1, r+1}^{(s)} & \ldots & l_{1, r+k}^{(s)} \\
\vdots & \vdots & \ldots & \vdots & \vdots & \vdots & \ldots & \vdots \\
0 & 0 & \ldots & l_{r r}^{(s)} & \vdots & l_{r, r+1}^{(s)} & \ldots & l_{r, r+k}^{(s)} \\
0 & 0 & \ldots & 0 & \vdots & 0 & \ldots & 0 \\
\vdots & \vdots & \ldots & \vdots & \vdots & \vdots & \ldots & \vdots \\
0 & 0 & \ldots & 0 & \vdots & 0 & \ldots & 0
\end{array}\right) .
$$

such that $r=\operatorname{rank}\left(L^{(0)}\right)$. If $k=n-r$, the solution to the coefficient of $d(x)$ is given as follows:

$$
\begin{aligned}
& d_{k-1}=l_{r, r+i}^{s} \operatorname{Inv}\left(l_{r r}^{(s)}\right) \\
& d_{k}=1
\end{aligned}
$$

for each $i=1,2, \ldots, k$. If $r=n$, then the degree of $d(x)=0$ which implies that GCD is a unit element.

\section{3-terms recurrence relation for the Chebyshev basis}

The shifted Chebyshev polynomials of the first kind is orthogonal defined by:

$$
T_{n}^{*}(x)=T_{n}(2 x-1)
$$

(12)

Where

$$
T_{n}^{*}(x)=\cos \left[n \cos ^{-1}(2 x-1)\right]=T_{n}(2 x-1)
$$


The recurrence relation for the polynomial is

$$
\begin{aligned}
& T_{0}^{*}(x)=1 \\
& T_{1}^{*}(x)=2 x-1 \\
& T_{n+1}^{*}(x)=(4 x-2) T_{n}^{*}(x)-T_{n-1}^{*}(x) \\
& \text { gives } \alpha_{0}=2, \beta_{0}=2, \alpha_{n}=4, \beta_{n}=-2, \gamma_{n}=1, \text { for } n=1,2, \ldots, n .
\end{aligned}
$$

Example 1:

Consider the polynomials in shifted chebyshev basis

and

$$
\begin{aligned}
a(x)= & p_{6}(x)-72 p_{5}(x)+1402 p_{4}(x)+3480 p_{3}(x) \\
& -299377 p_{2}(x)+1931952 p_{1}(x)-1637386
\end{aligned}
$$

$$
\begin{gathered}
b(x)=p_{5}(x)-6 p_{4}(x)-1875 p_{3}(x)+17976 p_{2}(x) \\
+485714 p_{1}(x)-501810
\end{gathered}
$$

With the value of each $\alpha, \beta$, and $\gamma$ as well as the coefficients of polynomial $a(x)$ known, the comrade matrix in (4) can be constructed as below,

$\left[\begin{array}{cccccc}0.5 & 0.5 & 0 & 0 & 0 & 0 \\ 0.25 & 0.5 & 0.25 & 0 & 0 & 0 \\ 0 & 0.25 & 0.5 & 0.25 & 0 & 0 \\ 0 & 0 & 0.25 & 0.5 & 0.25 & 0 \\ 0 & 0 & 0 & 0.25 & 0.25 & 0.25 \\ 409346 & -482988 & 74844.25 & -870 & -320.25 & 18.5\end{array}\right]$

From the comrade matrix, with the coefficients of polynomial $b(x)$ known, then the $b(A)$ matrix is constructed. By Theorem 1, the $b(A)$ matrix is rearranged so that a coefficient matrix is obtained i.e:

$\left[\begin{array}{llllll}1 & -6 & -1875 & 17976 & 485714 & -501810 \\ 34 & -1644 & 5370 & 409584 & -973084 & 559740 \\ 803 & -42258 & 291495 & 9193128 & -64643018 & 55199850 \\ 15524 & -831864 & 6351060 & 175638624 & -1430791544 & 1249618200 \\ 285061 & -15355806 & 120491745 & 3213894936 & -27252104806 & 23932788870 \\ 5153062 & -278046852 & 2200175790 & 58033455312 & -498213904852 & 438253167540\end{array}\right]$

The polynomials in this example is of degree 5 and 6 repectively. There is a large disparity in the sizes of the coefficients of the polynomials, considering monic polynomials wth coefficient of the highest degree terms equals 1 . The smallest entry of the matrix $b(A)$ is 1 while the largest entry has at least 15 digits. The matrix is ill conditioned. Preconditioning may be required in order to obtain a good approximate solution.

Let $\operatorname{deg}(a(x))=n$ and $d(x)=\operatorname{gcd}(a(x), b(x))$. Here, we have $k=\operatorname{deg}(d(x))=n-\operatorname{rank}[b(A)]$. In this paper, we consider the class of polynomials with big GCD in which case $k$ has to be at least $n / 2$, such that the coefficient matrix has small rank. We expect this to be the worst case, whereby roundoff errors are accumulated in the reduction and decomposition process.

The numerical methods considered in this work are Gauss Elimination with partial pivoting (GEPP), QR Householder decompositions and QR Householder with column pivoting (QRCP). Even though the GEPP method is not always stable, it is the simplest method as it can simultaneously determine the rank and reduces the coefficient matrix to its reduced form (11). From the rank, an augmented matrix representing the reduced systems of equations can be determined. Consequently, the desired coefficients of the GCD can be obtained directly from the reduced triangular matrix via backward substitutions. However, GEPP, is expected to be less appropriate and unstable for large matrices due to the accumulation of roundoff errors in the reduction process. The application of $\mathrm{QR}$ decomposition and QR with column pivoting which are known to be stable and widely used in solving the least square and over determined systems [12, 13, 14] are investigated and comparison between these algorithms is conducted.

\section{RESULTS AND DISCUSSION}

The test polynomials are generated by converting from power

\begin{tabular}{|c|c|}
\hline $\begin{array}{l}\text { Test } \\
\text { Examples }\end{array}$ & Polynomials relative to the Chebyshev basis \\
\hline $\begin{array}{l}\text { Test } 1 \\
\text { (degree } \\
a(x)=6, \\
\text { rank =2) }\end{array}$ & $\begin{array}{l}a(x) \\
=p_{6}(x)-72 p_{5}(x)+1402 p_{4}(x)+3480 p_{3}(x)-299377 p_{2}(x) \\
+1931952 p_{1}(x)-1637386 \\
b(x)=p_{5}(x)-6 p_{4}(x)-1875 p_{3}(x)+17976 p_{2}(x) \\
+485714 p_{1}(x)-501810 \\
\text { Greatest Common Divisor of } a(x) \text { and } b(x) \text { is } \\
d(x)=p_{4}(x)-48 p_{3}(x)+140 p_{2}(x)+12144 p_{1}(x)-12237\end{array}$ \\
\hline $\begin{array}{l}\text { Test } 2 \\
\text { (degree } \\
a(x)=6, \\
\text { rank=2) }\end{array}$ & $\begin{array}{l}a(x)=p_{6}(x)+128 p_{5}(x)+4794 p_{4}(x)+12416 p_{3}(x) \\
-2118385 p_{2}(x)-18444544 p_{1}(x)+86900790 \\
b(x)=p_{5}(x)+62 p_{4}(x)+125 p_{3}(x)-39672 p_{2}(x) \\
-184446 p_{1}(x)+3541690 \\
\text { Greatest Common Divisor of } a(x) \text { and } b(x) \text { is } \\
d(x)=p_{4}(x)+76 p_{3}(x)+1188 p_{2}(x)-23116 p_{1}(x) \\
-254629\end{array}$ \\
\hline $\begin{array}{l}\text { Test } 3 \\
\text { (degree } \\
a(x)=7, \\
\text { rank=2) }\end{array}$ & $\begin{array}{l}a(x)=p_{7}(x)+162 p_{6}(x)+9179 p_{5}(x)+190452 p_{4}(x) \\
-184215 p_{3}(x)-37744002 p_{2}(x)-4248645 p_{1}(x) \\
+528581868 \\
b(x)=p_{6}(x)+112 p_{5}(x)+3546 p_{4}(x)+3760 p_{3}(x) \\
-850801 p_{2}(x)+2699488 p_{1}(x)+3550614 \\
\text { Greatest Common Divisor of } a(x) \text { and } b(x) \text { is } \\
d(x)=p_{4}(x)+64 p_{3}(x)+380 p_{2}(x)-20544 p_{1}(x)+48259\end{array}$ \\
\hline $\begin{array}{l}\text { Test } 4 \\
\text { (degree } \\
a(x)=7, \\
\text { rank=3) }\end{array}$ & $\begin{array}{l}a(x)=p_{7}(x)+38 p_{6}(x)-1221 p_{5}(x)-59492 p_{4}(x) \\
+10825 p_{3}(x)+18622522 p_{2}(x)+89447035 p_{1}(x) \\
-548421628 \\
b(x)=p_{6}(x)+12 p_{5}(x)-1790 p_{4}(x)-19620 p_{3}(x) \\
+888047 p_{2}(x)+5696664 p_{1}(x)-72623602 \\
\text { Greatest Common Divisor of } a(x) \text { and } b(x) \text { is } \\
d(x)=p_{5}(x)+26 p_{4}(x)-1427 p_{3}(x)-39624 p_{2}(x) \\
+334738 p_{1}(x)+5211310\end{array}$ \\
\hline $\begin{array}{l}\text { Test } 5 \\
\text { (degree } \\
a(x)=7, \\
\text { rank=3) }\end{array}$ & $\begin{array}{l}a(x)=p_{7}(x)-42 p_{6}(x)+155 p_{5}(x)+11964 p_{4}(x) \\
-154071 p_{3}(x)+505482 p_{2}(x)+153915 p_{1}(x)-517404 \\
b(x)=p_{6}(x)-8 p_{5}(x)-822 p_{4}(x)+5400 p_{3}(x) \\
+141007 p_{2}(x)-1152272 p_{1}(x)+1006694 \\
\text { Greatest Common Divisor of } a(x) \text { and } b(x) \text { is } \\
d(x)=p_{4}(x)-16 p_{3}(x)-388 p_{2}(x)+3600 p_{1}(x)-3197\end{array}$ \\
\hline
\end{tabular}
series form such that the GCD of the polynomials is at least half the degree of the higher degree polynomial. In this paper, we present the

\begin{tabular}{|c|c|}
\hline $\begin{array}{c}\text { Test } 6 \\
\text { (degree } \\
\mathrm{a}(\mathrm{x})=8, \\
\text { rank=1) }\end{array}$ & $\begin{array}{l}a(x)=p_{8}(x)-24 p_{7}(x)-616 p_{6}(x)+15672 p_{5}(x) \\
+41692 p_{4}(x)-2092824 p_{3}(x)+8649256 p_{2}(x) \\
+2077176 p_{1}(x)-8690333 \\
b(x)=p_{7}(x)-18 p_{6}(x)-725 p_{5}(x) 11340+p_{4}(x) \\
\quad+110457 p_{3}(x)-1441422 p_{2}(x)-109733 p_{1}(x)+1430100 \\
\text { Greatest Common Divisor of } a(x) \text { and } b(x) \text { is } \\
d(x)=p_{7}(x)-18 p_{6}(x)-725 p_{5}(x)+11339 p_{4}(x) \\
+110457 p_{3}(x)-1441422 p_{2}(x)-109733 p_{1}(x)+1430100\end{array}$ \\
\hline $\begin{array}{c}\text { Test } 7 \\
(\text { degree } \\
\mathrm{a}(\mathrm{x})=8, \\
\text { rank=1) }\end{array}$ & $\begin{array}{l}a(x)=p_{8}(x)+44 p_{7}(x)-256 p_{6}(x)-31068 p_{5}(x) \\
-301332 p_{4}(x)+1035020 p_{3}(x)+11119936 p_{2}(x) \\
-1003996 p_{1}(x)-10818349 \\
b(x)=p_{7}(x)+26 p_{6}(x)-725 p_{5}(x)-18044 p_{4}(x) \\
+24185 p_{3}(x)+617734 p_{2}(x)-23461 p_{1}(x)-599716 \\
\text { Greatest Common Divisor of } a(x) \text { and } b(x) \text { is } \\
d(x)=p_{7}(x)+26 p_{6}(x)-725 p_{5}(x)-18044 p_{4}(x) \\
+24185 p_{3}(x)+617734 p_{2}(x)-23461 p_{1}(x)-599716\end{array}$ \\
\hline
\end{tabular}

results on the effects of partial pivoting in Gauss elimination, column pivoting in $\mathrm{QR}$ decomposition and normalization of the coefficient matrix columns on the condition number and the approximate solution of the GCD.

Table 1 Test examples of some polynomials (big GCD cases).

Table 2 Example of some polynomials (small rank, big GCD cases). 
The results of the implementation of the QRCP, QR Householder and GEPP algorithms on some test problems using $\mathrm{C}++$ programming are presented as shown in Table 3.

Table 3 Normwise relative error (big GCD cases).

\begin{tabular}{|c|c|c|c|}
\hline $\begin{array}{l}\text { Test } \\
\text { Examples }\end{array}$ & Method & $\begin{array}{l}\text { Before } \\
\text { normalization }\end{array}$ & $\begin{array}{l}\text { After } \\
\text { normalization }\end{array}$ \\
\hline \multirow{3}{*}{$\begin{array}{c}\text { Test } 1 \\
\text { (degree } \\
a(x)=6, \\
\text { rank=2) }\end{array}$} & QRCP & $1.54739 \times 10^{-5}$ & $1.43169 \times 10^{-7}$ \\
\hline & QR Householder & 0 & $1.49293 \times 10^{-7}$ \\
\hline & GEPP & 0 & $6.93148 \times 10^{-8}$ \\
\hline \multirow{3}{*}{$\begin{array}{l}\text { Test } 2 \\
\text { (degree } \\
a(x)=6, \\
\text { rank=2) }\end{array}$} & QRCP & $2.44221 \times 10^{-4}$ & $6.63630 \times 10^{-6}$ \\
\hline & QR Householder & $1.81654 \times 10^{-8}$ & $2.67883 \times 10^{-6}$ \\
\hline & GEPP & 0 & $2.21294 \times 10^{-6}$ \\
\hline \multirow{3}{*}{$\begin{array}{l}\text { Test 3 } \\
\text { (degree } \\
\mathrm{a}(\mathrm{x})=7, \\
\text { rank=2) }\end{array}$} & QRCP & 0.05187 & $8.93167 \times 10^{-5}$ \\
\hline & QR Householder & $4.36021 \times 10^{-4}$ & $8.62325 \times 10^{-5}$ \\
\hline & GEPP & 0 & $1.50610 \times 10^{-4}$ \\
\hline \multirow{3}{*}{$\begin{array}{l}\text { Test } 4 \\
\text { (degree } \\
a(x)=7, \\
\text { rank=3) }\end{array}$} & QRCP & $3.85379 \times 10^{-5}$ & $9.85492 \times 10^{-6}$ \\
\hline & QR Householder & 0 & $6.47758 \times 10^{-5}$ \\
\hline & GEPP & 0 & $7.00335 \times 10^{-5}$ \\
\hline \multirow{3}{*}{$\begin{array}{l}\text { Test } 5 \\
\text { (degree } \\
a(x)=7, \\
\text { rank=3) }\end{array}$} & QRCP & $1.34992 \times 10^{-5}$ & $1.19408 \times 10^{-6}$ \\
\hline & QR Householder & 0 & $9.95838 \times 10^{-7}$ \\
\hline & GEPP & 0 & $4.83029 \times 10^{-6}$ \\
\hline $\begin{array}{c}\text { Test } 6 \\
\text { (degree } \\
a(x)=8, \\
\text { rank=1) }\end{array}$ & QR Householder & 0 & $1.86584 \times 10^{-7}$ \\
\hline $\begin{array}{l}\text { Test } 7 \\
\text { (degree } \\
a(x)=8, \\
\text { rank=1) }\end{array}$ & QR Householder & 0 & $1.10550 \times 10^{-7}$ \\
\hline
\end{tabular}

${ }^{*}$ QRCP - Qr Householder with column pivoting

${ }^{*}$ GEPP - Gauss Elimination with partial pivoting

Table 4 : Condition number of associated coefficient matrix.

\begin{tabular}{|c|c|c|}
\hline Test examples & $\begin{array}{c}\text { Before } \\
\text { normalization }\end{array}$ & After normalization \\
\hline Test 1 & $1.58796 \mathrm{e}+024$ & $2.06467 \mathrm{e}+013$ \\
\hline Test 2 & $2.41847 \mathrm{e}+025$ & $7.46846 \mathrm{e}+013$ \\
\hline Test 3 & $1.23251 \mathrm{e}+027$ & $1.20487 \mathrm{e}+013$ \\
\hline Test 4 & $1.05173 \mathrm{e}+027$ & $4.28692 \mathrm{e}+013$ \\
\hline Test 5 & $2.50945 \mathrm{e}+023$ & $9.06619 \mathrm{e}+006$ \\
\hline Test 6 & $1.83353 \mathrm{e}+084$ & $1.96320 \mathrm{e}+066$ \\
\hline Test 7 & $6.32532 \mathrm{e}+045$ & $2.27361 \mathrm{e}+063$ \\
\hline
\end{tabular}

The norm-wise relative error results in Table 3, illustrated that for test examples $1-5$, the algorithms give a good approximation to the respective solution with reasonably small relative error, even though the condition number of the respective coefficient matrix indicate that the computed solutions ought to be sensitive to the order of arithmetic and precision applied. For each of these test examples, GEPP and QR Householder produce a better approximate solution compared to QRCP which shows that partial pivoting in the GEPP and QR without pivoting is sufficient to give a good approximate solution. As shown in Table 4, for all the test examples, the condition number for each coefficient matrix is slightly reduced after each column is normalized so as to have a uniform size in the column entries, in which case the QRCP do gives a better approximated solution after normalization. Thus for QRCP equilibration of the columns by $\infty$-norm reduces the condition number and is also able to improve the solution.

Test examples 6 and 7 consider the cases whendeg $(a(x))=8$, such that the rank of $b(A)$ equals 1

Thus the degree of the GCD is 7, which is just one degree less than that of degree $a(x)$. For these polynomials, only $\mathrm{QR}$ Householder is able to give a good approximate solution. GEPP and QRCP fail to give a good approximation. These two examples are severely ill-conditioned compared to the other test examples. It can be seen for these two cases that normalizing each column of the coefficient matrix does not contribute a significant effect in reducing the condition number of the matrix. Nor does it lead to a good approximate solution, with the exception of the $\mathrm{QR}$ algorithm which proves to remain stable.

\section{CONCLUSION AND FURTHER WORK}

The results reveal the potential of the Gauss elimination with partial pivoting to solving overdetermined systems of linear equations up to a certain degree, despite the ill-condition property of the matrix involved, as depicted in the varying magnitude of the entries of the matrix and its respective condition number. The empirical results in the norm-wise relative error shows that the GEPP method is as competitive as the $\mathrm{QR}$ algorithm with column pivoting. The latter method involves finding the inverse of a triangular matrix before the final solution is obtained. On the other hand, QR Householder has less complexity, without having to do column pivoting and computing matrix inverse. It is the most effective method, so far. Further work is to work on test examples 6 and 7 and higher degree polynomials. Appropriate preconditioning or preprocessing strategies has to be determined since the higher the degree of the polynomials, the size of the integer matrix entries will also increase. Consequently, the larger will be the disparity in the magnitude of the entries of the matrix involved.

\section{ACKNOWLEDGEMENT}

This work was financially supported by the Universiti Teknologi Malaysia under the Research University Grant and Ministry of Higher Education Malaysia, GUP grant vot $12 \mathrm{~J} 30$.

\section{REFERENCES}

[1] B. Liang, S. U. Pillai. (1997). Two-dimensional blind deconvolution using a robust GCD approach. Proceedings of the 1997 International Conference on Image Processing. Part 2 (of 3). 26-29 October. Santa Barbara, CA, USA: IEEE, 424-427.

[2] S. U. Pillai, B. Liang. (1999). Blind image deconvolution using a robust GCD Approach. IEEE Transactions on Image Processing 8(2), 295-301.

[3] P.Stoica, T. Söderström, (1997). Common factor detection and estimation. Automatica, 33(5), 985-989.

[4] Z. Zheng. (2005). Computing multiple roots of inexact polynomials. Mathematics of Computation, 74, 869-903.

[5] S. Barnett. (1975). A companion matrix analogue for orthogonal polynomials. Linear Algebra and its Applications 12(3), 197-202.

[6] s. barnett. (1971). greatest common divisor of several polynomials. Mathematical Proceedings of the Cambridge Philosophical Society, (Cambridge University Press, 1971), 70, 263-268.

[7] S. Barnett. (1983). Polynomials and Linear Control Systems Marcel. New York, NY, USA: Dekker, Inc.

[8] N. Aris, A. A. Rahman. (2001). On the computation of the GCD of polynomials relative to an orthogonal basis," Technical Report LT/M Bil.1/2001, April 2001.

[9] N. Aris, S. N. Ahmad. (2008). Computing the greatest common divisor of polynomials using the comrade matrix. In D. Kapur (Ed.), Computer Mathematic, 87-96. Heidelberg: Springer Berlin Heidelberg.

[10] N. Aris. (2003). On the application of modular approach to the computation of the greatest common divisor of generalized polynomials. Doctoral Thesis. Universiti Teknologi Malaysia.

[11] S. N. A. Isa, N. Aris, S. M. Puzi, in N. Rusli, W. M. K. A. W. Zaimi, K. A. M. Khazali, M. J. Masnan, W. S. W. Daud, N. Abdullah, \& N. A. M. Amin (Eds.). (2016). Numerical matrix methods in the computation of the greatest common divisor (GCD) of polynomials. AIP Conference Proceedings, 1775(1), 030064).

[12] B. N. Datta. (2010) Numerical linear algebra and applications. India: Prentice Hall.

[13] G. H. Golub, C. F. Van Loan. (2012). Matrix computations ( ${ }^{\text {rd }}$ Edition). USA: Johns Hopkins University Press.

[14] N. J. Higham. (2002). Accuracy and stability of numerical algorithms $\left(2^{\text {nd }}\right.$ Edition). Philadelphia: Society for Industrial and Applied Mathematics (SIAM). 